\title{
Neutralization of IL-8 decreases tumor PMN-MDSCs and reduces mesenchymalization of claudin-low triple-negative breast cancer
}

\author{
Charli Dominguez, Kristen K. McCampbell, Justin M. David, and Claudia Palena \\ Laboratory of Tumor Immunology and Biology, Center for Cancer Research, National Cancer Institute, NIH, Bethesda, \\ Maryland, USA.
}

The complex signaling networks of the tumor microenvironment that facilitate tumor growth and progression toward metastatic disease are becoming a focus of potential therapeutic options. The chemokine IL-8 is overexpressed in multiple cancer types, including triple-negative breast cancer (TNBC), where it promotes the acquisition of mesenchymal features, stemness, resistance to therapies, and the recruitment of immune-suppressive cells to the tumor site. The present study explores the utility of a clinical-stage monoclonal antibody that neutralizes IL-8 (HuMax-IL8) as a potential therapeutic option for TNBC. HuMax-IL8 was shown to revert mesenchymalization in claudin-low TNBC models both in vitro and in vivo as well as to significantly decrease the recruitment of polymorphonuclear myeloid-derived suppressor cells (PMN-MDSCs) at the tumor site, an effect substantiated when used in combination with docetaxel. In addition, HuMax-IL8 enhanced the susceptibility of claudin-low breast cancer cells to immune-mediated lysis with NK and antigen-specific $T$ cells in vitro. These results demonstrate the multifaceted way in which neutralizing this single chemokine reverts mesenchymalization, decreases recruitment of MDSCs at the tumor site, assists in immune-mediated killing, and forms the rationale for using HuMax-IL8 in combination with chemotherapy or immune-based therapies for the treatment of TNBC.

Conflict of interest: The authors have declared that no conflict of interest exists.

Submitted: March 28, 2017 Accepted: September 26, 2017 Published: November 2, 2017

\section{Reference information:} JCI Insight. 2017;2(21):e94296. https://doi.org/10.1172/jici. insight.94296.

\section{Introduction}

Breast cancer is the most commonly diagnosed cancer in women (1); it presents with a diverse phenotype and considerable heterogeneity in molecular and histopathological features. Accounting for 1 in 10 instances of newly diagnosed breast cancers, triple-negative breast cancer (TNBC) is defined by the absence of estrogen and progesterone receptor expression and the lack of human epidermal growth factor receptor-2 (HER2) overexpression and/or amplification $(2,3)$. Within TNBC, several molecular subtypes have been identified, with the two predominant groups corresponding to the basal-like and claudin-low subtypes $(4,5)$. Claudin-low breast tumors, which account for approximately $30 \%$ of TNBC cases, are characterized by traits of carcinoma mesenchymalization, i.e., high expression of mesenchymal proteins and low expression of epithelial cell junctions, and a marked lymphocytic infiltration. Moreover, several studies have now demonstrated the presence of breast stem cell-like cells in claudin-low tumors, characterized by the expression of aldehyde dehydrogenase 1 (ALDH1) and the phenotype $\mathrm{CD} 44^{+} \mathrm{CD} 24^{-/ 10}(6)$. Both tumor stemness and mesenchymalization have been proposed to contribute to the highly aggressive and metastatic nature of claudin-low breast cancer, due to the roles that these phenomena play in promoting therapy resistance and tumor dissemination.

In the absence of expression of hormone and HER-2 receptor and having features of tumor stemness, TNBC remains a disease refractory to advancements in targeted and conventional therapies (7), posing the need for novel therapeutic options. A central feature in cancer is the presence of relevant signaling networks established between the cancer cells and the various components of the tumor microenvironment, including soluble mediators such as growth factors, chemokines, and cytokines. One of the soluble factors that has gained attention in the field of cancer progression is the chemokine IL-8 (CXCL8). Initially, IL-8 was identified for its role in the chemoattraction of neutrophils in the context of infection and inflammation $(8,9)$. 
However, abnormally high expression of IL-8 has been observed in multiple cancers (10), including in breast tumors (11). IL-8 signaling has been shown to foster tumor progression by various means, including (a) promoting the trafficking of immune-suppressive cells to the tumor microenvironment (12); (b) promoting tumor mesenchymalization $(13,14)$; and (c) increasing the survival of cancer stem-like cell populations in the tumor (15). IL-8 signaling has also been shown to stimulate the recruitment into the tumor of myeloid-derived immune suppressor cells (MDSCs), which exert a suppressive role on antitumor immunity (16).

The multifaceted roles of IL- 8 in cancer make it a promising therapeutic target. Blockade of IL- 8 signaling could modulate tumor phenotype, reduce recruitment of immune-suppressive cells to the tumor site, and inhibit cancer stem-like cells, thereby attacking multiple avenues that contribute to tumor progression and treatment resistance with a single approach. While there have been reports employing this tactic with IL- 8 receptor blockade both in the preclinical and clinical settings (15-18), to our knowledge this study is the first to utilize an IL-8-specific antibody to target IL-8 signaling in a preclinical model of claudin-low TNBC. Here, the high-affinity, fully human, clinical-stage monoclonal anti-IL-8 antibody, termed HuMax-IL8 (19, 20 ), was explored both in vitro and in vivo with xenografts of claudin-low breast cancer cells. These studies demonstrated the ability of an anti-IL-8 monoclonal antibody to (a) reduce mesenchymal features in cancer cells; (b) reduce the frequency of polymorphonuclear MDSCs (PMN-MDSCs) found at the tumor site, an effect that was enhanced when HuMax-IL8 was combined with docetaxel treatment in MDA-MB-231 xenografts; and (c) enhance the susceptibility of claudin-low TNBC cells to lysis mediated by immune effector $\mathrm{NK}$ and antigen-specific $\mathrm{T}$ cells in vitro. Altogether, these data provide the rationale for combination therapies involving the use of HuMax-IL8 antibody with chemotherapy or immune-based therapies in the claudin-low breast cancer group and, potentially, in other tumors driven by an IL-8 autocrine loop.

\section{Results}

Eight TNBC cell lines with different phenotypes were screened for levels of IL-8 secretion and used to assess the effect of autocrine IL-8 signaling blockade with the anti-IL-8 monoclonal antibody, HuMax-IL8. Of these cell lines, four have been previously characterized (21) as claudin-low (BT549, Hs578T, MDAMB-436, and MDA-MB-231), two as basal like (HCC1143 and SUM149PT), and two as luminal (MCF7 and ZR-75-1). Each of the claudin-low and basal-like cell lines secreted characteristically high levels of IL-8, while the two luminal lines produced minimal to no IL-8 in culture (Figure 1A). As shown in Figure 1A, HuMax-IL8 markedly reduced the levels of free IL-8 in all claudin-low and basal-like cell lines, an effect that resulted in a significant reduction of proliferation in the basal-like cells (Figure 1B), which was not observed with any of the claudin-low cells. These results were in agreement with a previous report that demonstrated that autocrine IL-8 signaling is needed for the growth of basal-like but not claudin-low breast cancer cells (17). Based on these results, we subsequently focused our investigations on the claudinlow subtype of breast cancer to elucidate whether IL-8 blockade could affect tumor features other than cell proliferation, including expression of various epithelial and mesenchymal markers. Interestingly, HuMaxIL8 treatment of claudin-low cells markedly enhanced the expression of CXCR2 in 3 of 4 lines evaluated (BT549, Hs578T, and MDA-MB-231, Figure 1C), while CXCR1 was downregulated in the same cell lines. Since the IL-8 receptor CXCR2 is also able to bind other chemokines of the C-X-C family, the expression of cytokines and chemokines other than IL-8 was also assessed in tumor cells treated with HuMaxIL8. A cytokine array performed with supernatants obtained from MDA-MB-231 and MDA-MB-436 cells exposed to HuMax-IL8 in culture demonstrated a decrease in free IL-8 without any effect on CXCL5, GRO $-\alpha / \beta / \gamma$, CXCL6, or CXCL7 (Supplemental Figure 1; supplemental material available online with this article; https://doi.org/10.1172/jci.insight.94296DS1).

Considering that a central feature of claudin-low TNBCs is the predominant expression of mesenchymal versus epithelial proteins, and given the known role of IL-8 in driving carcinoma mesenchymalization in several tumor types (22-24), the potential reversion of tumor phenotype upon neutralization of IL-8 with HuMax-IL8 was explored both in vitro and in vivo with the claudin-low cell lines MDA-MB-231 and MDAMB-436. In vitro, a panel of epithelial-to-mesenchymal transition (EMT) markers was evaluated at the mRNA level in cells treated with HuMax-IL8 versus control IgG. Neutralization of IL-8 resulted in a marked increase in mRNAs encoding for epithelial makers, including the tight junction protein ZO-1, E-cadherin, and occludin, coupled with a significant reduction in the expression of mRNAs encoding for the mesenchymal markers fibronectin, vimentin, snail, or twist1 (Figure 2A). To further substantiate the observed changes in EMT, expression of epithelial E-cadherin and mesenchymal fibronectin was evaluated via immunofluorescence. 
A

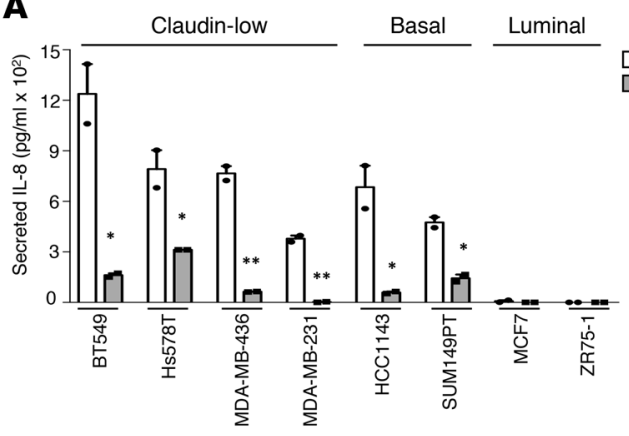

B

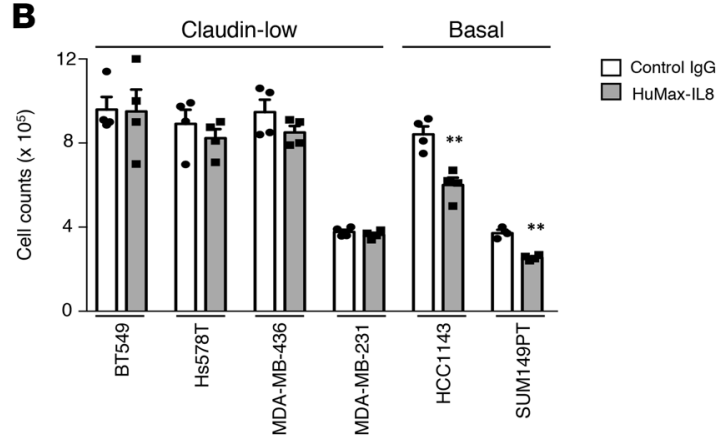

C
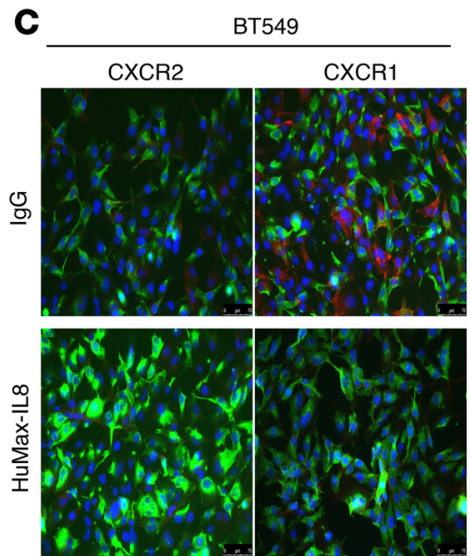

\begin{tabular}{cc} 
Hs578T \\
\hline CXCR2
\end{tabular}
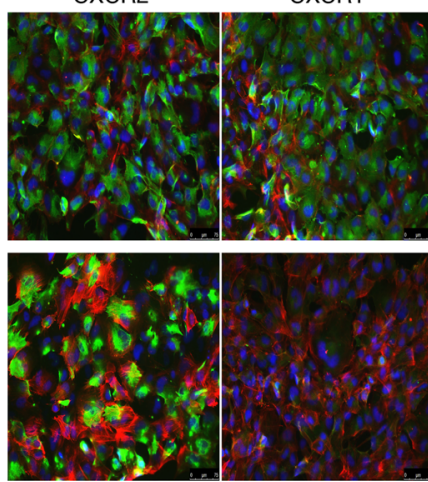
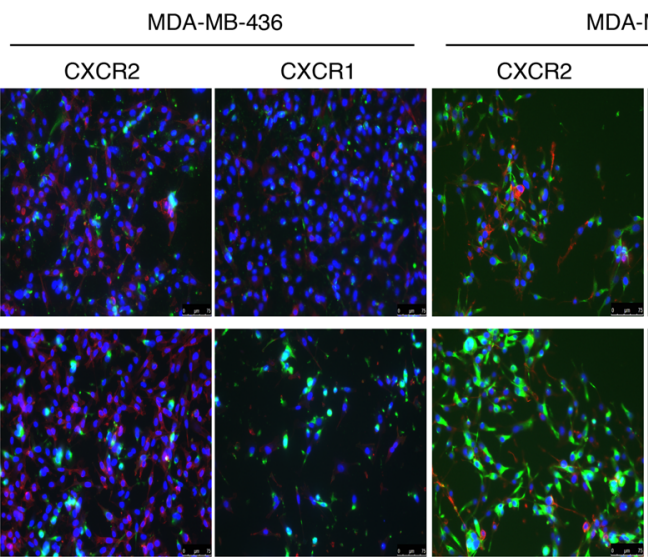

MDA-MB-231

CXCR1

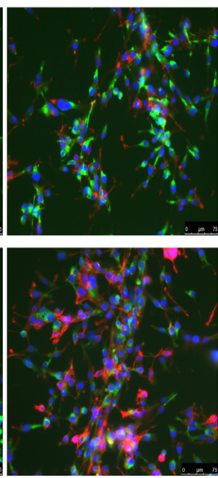

Figure 1. HuMax-IL8 neutralizes free IL-8 released by TNBC cells. (A) Assessment of secreted levels of IL-8 in culture supernatants of indicated breast cancer cell lines treated with HuMax-IL8 versus control IgG $(25 \mu \mathrm{g} / \mathrm{ml})$ for 3 days. Data represent mean (bars) $+\mathrm{SEM}$ (error bars); $n=2$ (dots); differences between means were compared using 2-tailed unpaired $t$ test for each cell line; ${ }^{*} P<0.05$; ${ }^{*} P<0.01$. (B) Cell counts of indicated tumor cell lines treated with HuMax-IL8 versus control IgG $(25 \mu \mathrm{g} / \mathrm{ml}$ ) for 3 days. Data represent mean (bars) + SEM (error bars); $n=4$ (dots); differences between means were compared using 2-tailed unpaired $t$ test for each cell line; ${ }^{*} P<0.05$; ${ }^{*} P<0.01$. (A and $\mathbf{B}$ ) Data are representative of 3 experiments for the claudin-low lines and 1 experiment for the basal and luminal lines. (C) Immunofluorescent detection of CXCR1 and CXCR2 expression in indicated cell lines. Blue: DAPIstained nuclei; red: phalloidin staining; green: CXCR1 or CXCR2, as indicated. Original magnification, $\times 20$; scale bars: $75 \mu \mathrm{m}$.

As shown in Figure 2B, neutralization of IL-8 induced expression of E-cadherin and a decrease in fibronectin expression in both cell lines, which resulted in an increase of the E-cadherin/fibronectin ratio from 0.4 to 36.5 and from 0.2 to 3.6 for the IgG versus HuMax-IL8-treated MDA-MB-231 and MDA-MB-436 cells, respectively (Figure 2B). Similar results were seen with BT549 cells (Supplemental Figure 2).

The role of IL-8 neutralization on the phenotype of claudin-low breast cancer cells was further analyzed in vivo with MDA-MB-231 cells grown as xenografts in the mammary fat pad of C.B-17 SCID mice. Following two administrations of control human IgG versus HuMax-IL8 at $200 \mu \mathrm{g} / \mathrm{mouse}$ every 2 days, tumors were harvested and IHC was conducted for various phenotypic markers. Upon depletion of tumorderived human IL-8 in vivo, a marked increase of the epithelial markers E-cadherin and ZO-1, along with a reduction of mesenchymal vimentin and fibronectin, was observed in HuMax-IL8- (T3 and T4, Figure 3 versus control IgG-treated (T1 and T2, Figure 3) tumors (see Figure 3 for two representative tumors in each group). These differences were confirmed by capturing digital images of each stained tissue followed by quantification of the positive IHC signal, as described in the Methods and shown in the right panels of Figure 3. In addition, expression of the stemness-associated marker ALDH1A1, previously shown to be upregulated in breast cancer stem cells $(15,25)$, was markedly decreased in MDA-MB-231 xenografts from mice treated with HuMax-IL8 (T3 and T4, Figure 3) versus control IgG (T1 and T2, Figure 3). These data substantiate the role of IL-8 in tumor mesenchymalization and acquisition of stemness features, indicating the ability of HuMax-IL8 to revert tumor phenotype toward a more epithelial-like state.

It has been previously shown that MDSC populations expand in athymic mice bearing TNBC xenografts, specifically MDA-MB-231, during tumor development (26). The chemotactic role of IL-8 toward immune cell populations includes the ability of this chemokine to recruit MDSCs to the tumor microenvironment $(27,28)$. Although there is no homolog of IL-8 in the mouse genome, the IL- 8 receptor CXCR2 
A

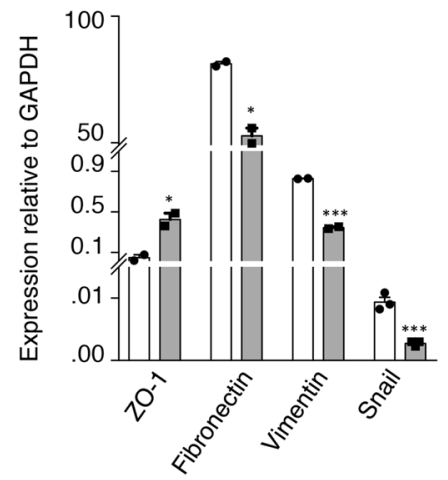

MDA-MB-436

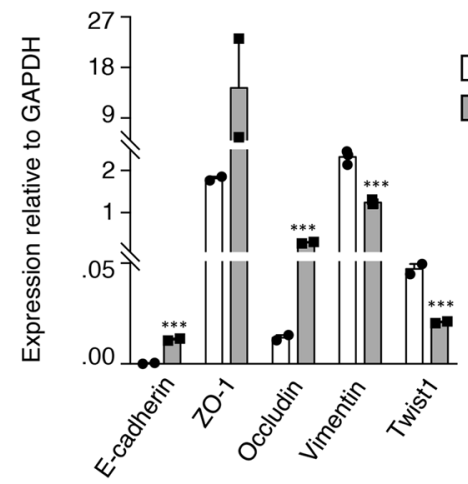

Control lgG

HuMax-IL8
B

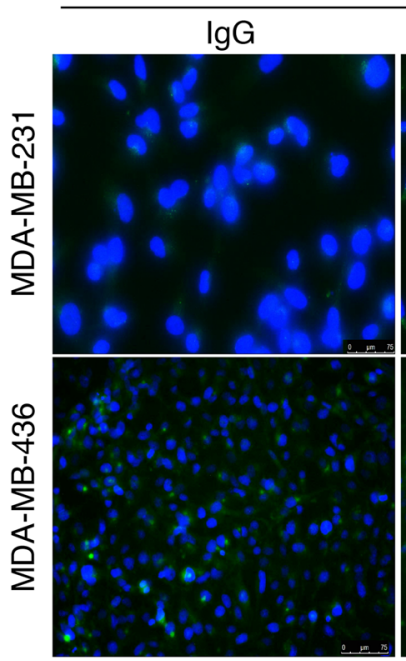

E-cadherin

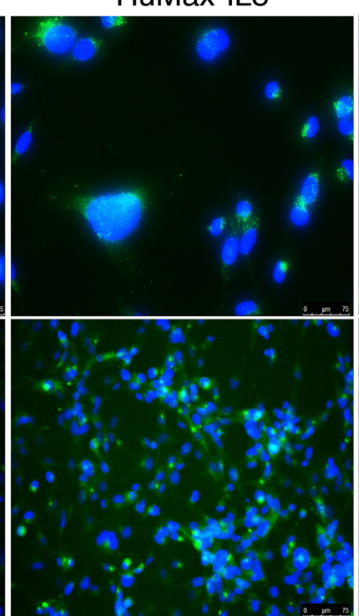

Fibronectin

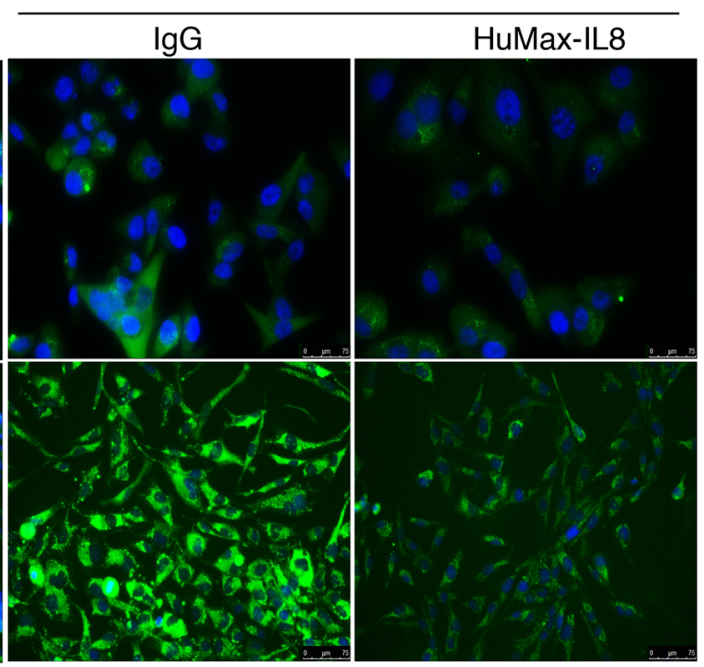

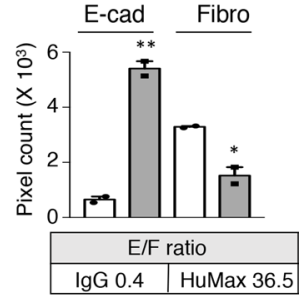

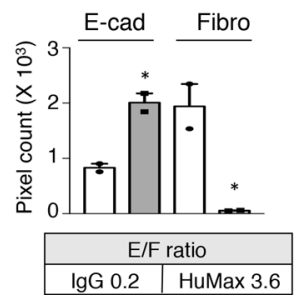

Figure 2. HuMax-IL8 reduces mesenchymalization of claudin-low breast cancer cells in vitro. Indicated tumor cell lines were treated with control IgG or HuMax-IL8 (25 $\mu \mathrm{g} / \mathrm{ml}$ ) for 3 days. (A) mRNA expression of indicated EMT markers in MDA-MB-231 and MDA-MB-436 cells (data are representative of 3 experiments). (B) Immunofluorescent analysis of E-cadherin and fibronectin expression (green); blue: DAPI-stained nuclei. Original magnification: $\times 20$; scale bars: $75 \mu \mathrm{m}$. Graphs correspond to the quantification of green fluorescence (E-cadherin and fibronectin, respectively, utilizing ImageJ binary pixel intensity analysis). The ratio of E-cadherin/fibronectin (E/F) expression in IgG-versus HuMax-IL8-treated cells is shown below. In all graphs, data represent mean (bars) + SEM (error bars) from $n=2-3$ experimental replicates (dots); differences between means were compared using 2-tailed unpaired $t$ test for each cell line; ${ }^{*} P<0.05 ;{ }^{*} P<0.01 ;{ }^{* *} P<0.001$.

is expressed in murine MDSCs, and we hypothesize that human IL-8 secreted by MDA-MB-231 cancer cells could bind murine CXCR2 and elicit the recruitment of MDSCs into the tumor. The chemoattractant properties of human IL-8 on these cells was explored in vitro; as shown in Figure 4A, murine CD11b cells migrated significantly toward media containing recombinant human IL-8, an effect that was completely abrogated by HuMax-IL8. In order to more accurately assess the chemoattractant capability of IL-8 produced by human cancer cells, MDA-MB-231 cells were pretreated with IgG or HuMax-IL8, and the culture media were used in a chemotaxis assay. The migration of $\mathrm{CD} 11 \mathrm{~b}^{+}$cells was significantly reduced with culture media from cells pretreated with HuMax-IL8 versus IgG (Figure 4B). These results indicated that human IL- 8 has the ability to chemoattract murine CD11b cells.

C.B-17 SCID mice bearing MDA-MB-231 xenografts were utilized to evaluate the influence of HuMaxIL8 treatment on tumor recruitment of murine MDSCs, both as a monotherapy and in combination with chemotherapy. Upon tumor establishment, mice were administered HBSS, docetaxel, HuMax-IL8, or the combination of docetaxel and HuMax-IL8, as specified in the Methods. As shown in Figure 5, A and B, a reduction of tumor volume was observed with docetaxel or HuMax-IL8 treatment alone, though the antitumor effect was more pronounced in the combination group. Analysis of human IL- 8 in the sera of these mice showed a noticeable enhancement of circulating IL- 8 in the docetaxel group, an observation attributed to the known effect of chemotherapy treatment on tumor IL- 8 secretion due to activation of stress-activated protein kinases 


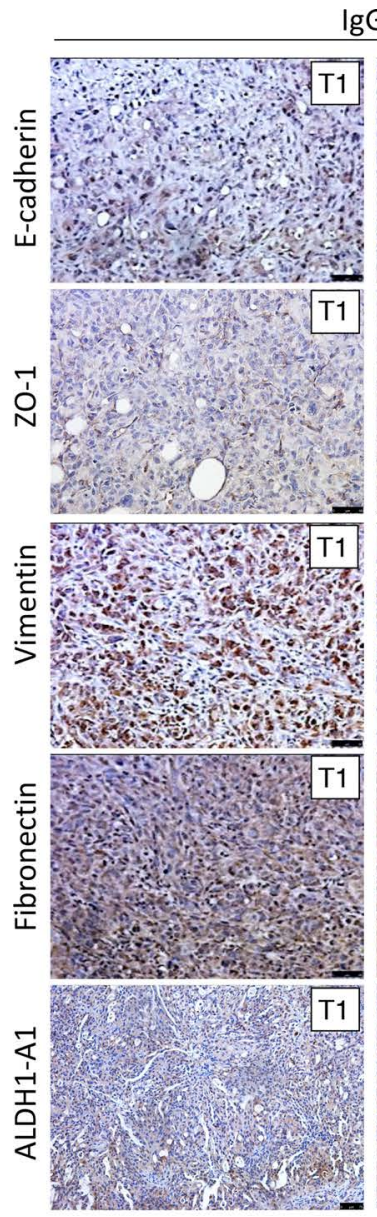

IgG

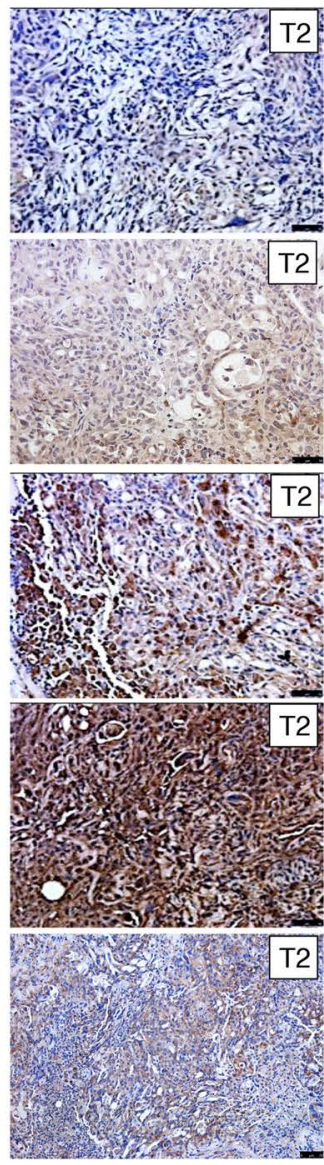

HuMax-IL8
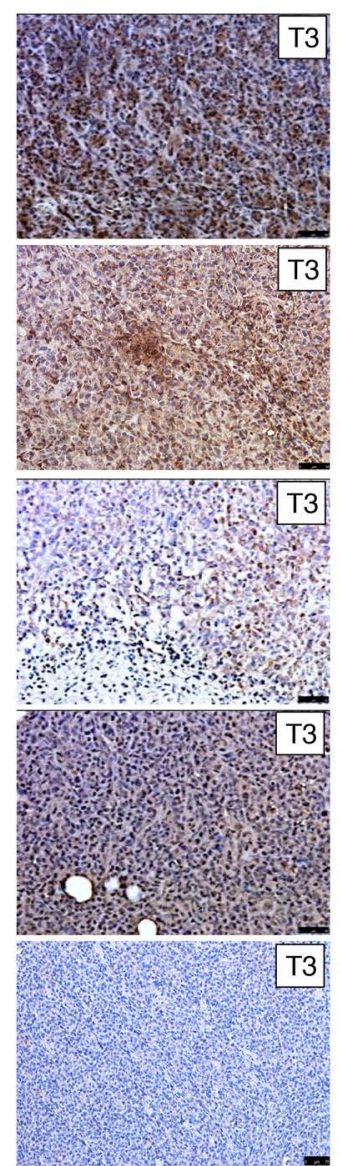

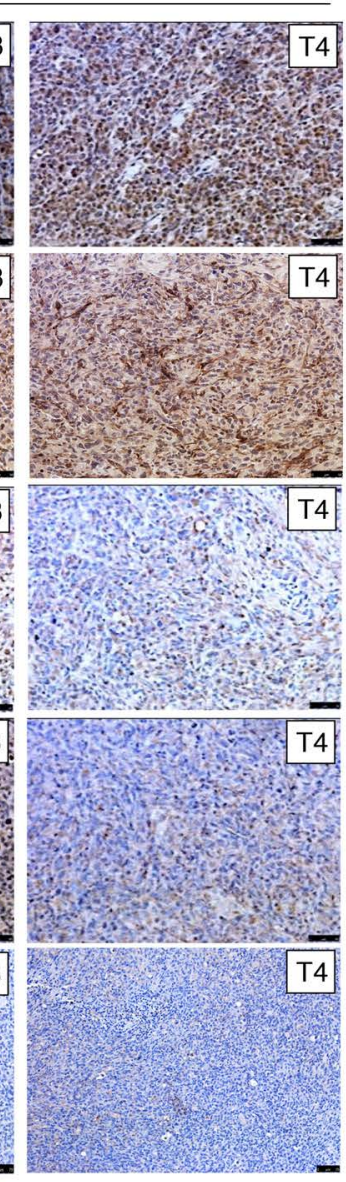

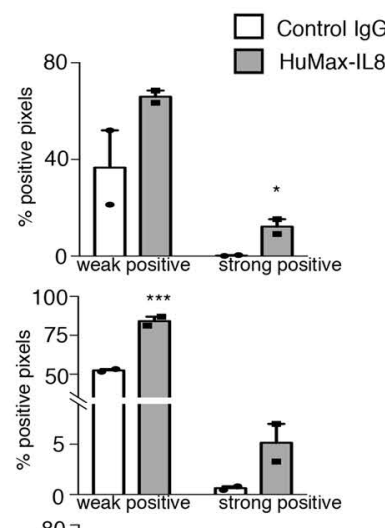

807
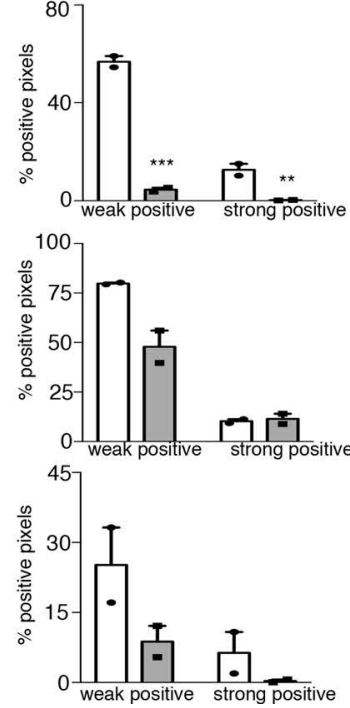

Figure 3. Neutralization of IL-8 reduces features of mesenchymalization and stemness in vivo. Representative images of MDA-MB-231 xenografts depicting the expression levels of the epithelial markers E-cadherin and ZO-1, the mesenchymal proteins fibronectin and vimentin, and the stemness marker ALDH1A1, as determined by IHC. Original magnification, $\times 20$; scale bars: $75 \mu \mathrm{m}$. Slides were digitally scanned with an Aperio ScanScope scanning system (Aperio Technologies Inc.) and analyzed by using the Aperio ImageScope Viewer software. The positive pixel count algorithm was used to measure the intensity of each marker (brown signal). Weak and strong positive staining was recorded for the whole tumor section, and the percentage of weak and strong positive pixels was calculated relative to the total number of pixels in the section. T1 and T2 are two representative tumors from mice treated with control lgG; T3 and T4 correspond to two representative tumors from mice treated with HuMax-IL8. Graphs represent mean (bars) + SEM (error bars) from $n=2$ representative tumors (dots) in each treatment group; differences between means were compared using 2-tailed unpaired $t$ test for each marker; ${ }^{*} P<0.05$; ${ }^{* *} P<0.01$; ${ }^{* * *} P<0.001$. The experiment was repeated 2 times with $n=5$ tumors per group.

and NF-кB $(29,30)$. The combination of HuMax-IL8 with chemotherapy, however, efficiently reduced the levels of circulating, free human IL-8, thus demonstrating the ability of HuMax-IL8 to bind and neutralize human IL-8 in vivo (Figure 5C). Analysis of immune cell populations at the tumor site demonstrated a significant enhancement of the percentage of $\mathrm{CD}_{11} \mathrm{~b}^{+}$cells in the docetaxel and combination groups, compared with the control group (Figure 5D). A phenotypic characterization of MDSC populations, however, showed a reduction in infiltrating PMN-MDSCs, which are defined by the phenotype CD $11 b^{+}$Ly $_{6} \mathrm{G}^{\text {hi }} / \mathrm{Ly} 6 \mathrm{C}^{\mathrm{lo}}$ in the docetaxel and HuMax-IL8 monotherapy groups. An even more significant reduction was observed when docetaxel and HuMax-IL8 were given in combination (Figure 5, D and E). This decrease in PMN-MDSCs at the tumor site was also accompanied by a significant increase in the percentage of monocytic MDSCs (M-MDSCs), defined as CD11b $\mathrm{Ly}_{6} \mathrm{G}^{\mathrm{lo} /-} / \mathrm{Ly} 6 \mathrm{C}^{\mathrm{hi}}$, in both the HuMax-IL8 alone and combination groups (Figure 5, D and E). The findings at the tumor site did not correspond with changes in the spleen or bone marrow. As shown in Figure 6, A and B, no changes in any of the treatment groups were seen in the spleen. In the bone marrow, a slight increase was observed with $\mathrm{CD} 11 \mathrm{~b}^{+}$cells in the combination group, and a minor but significant decrease of PMN-MDSCs was seen in the monotherapy groups (Figure 6, C and D). These findings revealed the ability of HuMax-IL8 to diminish the infiltration of granulocytic PMN-MDSCs at the tumor site, an effect that was particularly prominent when used in combination with chemotherapy. 
A $\quad$ CD11 $\mathrm{b}^{+}$Murine Cells

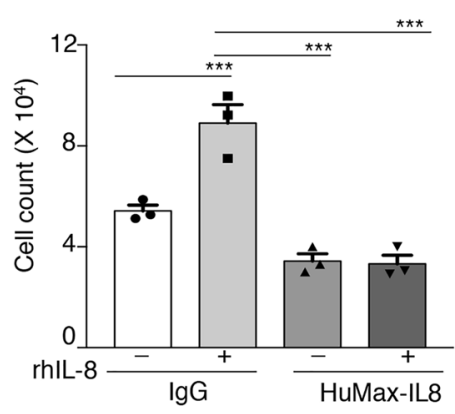

B

CD11 $b^{+}$Murine Cells

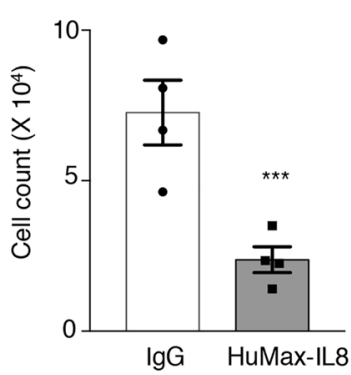

Figure 4. Neutralization of IL-8 reduces chemotaxis of murine CD11b ${ }^{+}$ cells. (A) Cell counts of murine CD11 $\mathrm{b}^{+}$chemotaxis induced via media containing IgG $(25 \mu \mathrm{g} / \mathrm{ml})$ or HuMax-IL8 $(25 \mu \mathrm{g} / \mathrm{ml})$, with or without addition of recombinant human IL-8 $(100 \mathrm{ng} / \mathrm{ml})$. Data represent mean (bars) + SEM (error bars) from $n=3$ (dots); differences between means were compared using 1-way ANOVA with Tukey's multiple comparisons test; ${ }^{* *} P<0.001$. Data are representative of 3 experiments. (B) Chemotaxis of murine $\mathrm{CD}_{11 \mathrm{~b}^{+}}$cells in response to cultured media obtained from MDA-MB-231 cells pretreated with IgG or HuMax-IL8. Data represent mean (bars) + SEM (error bars) from $n=4$ (dots); differences between means were compared using a 2-tailed unpaired $t$ test; ${ }^{* * *} P<0.001$. Data are representative of 2 experiments.

The modulation of tumor phenotype by HuMax-IL8 treatment was also investigated in the combination study. Control- and docetaxel-treated tumors exhibited a predominant mesenchymal phenotype, with high expression of vimentin. Upon neutralization of tumor-derived human IL-8, a substantial reduction of vimentin expression and a slight increase of E-cadherin were observed in both the monotherapy and combination groups (Supplemental Figure 3). These results agreed with the prior observations in the monotherapy study (Figure 3).

The effective reduction of PMN-MDSCs mediated by HuMax-IL8 treatment raises the potential for using this agent in combination with immune-based therapies. To elucidate the possible pairing of HuMaxIL8 antibody with immunotherapy, TNBC cell lines were pretreated in vitro with HuMax-IL8 prior to being used as targets in cytotoxic lytic assays with immune effector cells. Initially, NK cells isolated from healthy donors were used as effector cells. HuMax-IL8 pretreatment markedly increased NK-mediated lysis of all 4 cell lines evaluated to levels above those observed with control IgG-treated tumor cells (Figure 7A). Similarly, a significant enhancement of antigen-specific T cell-mediated lysis of MDA-MB-231 cells was observed when utilizing HLA-A ${ }_{02}$-restricted MUC1-, CEA-, and brachyury-specific T cell lines generated from the blood of cancer patients, as described in the Methods (Figure 7B). Since the enhancement of immune-mediated lysis was seen with both antigen-independent $(\mathrm{NK})$ and antigen-specific immune cells, the ability of HuMax-IL8 to improve general apoptotic responses in TNBC cells was investigated. MDAMB-231, Hs578T, and MDA-MB-436 cells were pretreated with the pan-caspase inhibitor Z-VAD-FMK, following pretreatment with HuMax-IL8; subsequently, they were used as targets for NK-mediated lysis or for killing by the extrinsic apoptotic trigger, TRAIL. With all cell lines, the enhancement of tumor lysis seen with HuMax-IL8 treatment in response to both NK cells and TRAIL was completely abrogated by the blockade of caspase activity (Figure 7C), therefore indicating that HuMax-IL8 increases the general susceptibility of TNBC cells to caspase-mediated apoptosis. Additionally, the role of perforin/granzymedependent lysis was explored in this system utilizing antigen-specific $\mathrm{T}$ cells pretreated with concanamycin A (CMA), which abrogates the activity of perforin and granzyme proteins in the effector cells. The significant increase in lysis observed with HuMax-IL8-treated cancer cells was only partially decreased by inhibition of the perforin/granzyme lytic pathway (Figure 7D), thus indicating that HuMax-IL8-treated TNBC cells became more susceptible to lysis by immune cells even in the absence of functional perforin/ granzyme. The improvement of immune-mediated lysis following HuMax-IL8 treatment was also found to be independent of any changes in the expression of MHC class I, costimulatory, or inhibitory molecules, as no changes were observed in HLA-ABC, MICA, CD54, CD58, CD80, or PD-L1 expression (Supplemental Table 1). However, increased expression of several molecules known to participate in antigen processing and presentation, including TAP1, TAP2, and Tapasin, and increased expression of the surface-associated death receptors TRAIL-R1 and TRAIL-R2 were observed in MDA-MB-231 cells following HuMax-IL8 treatment (Supplemental Figure 4). Both groups of molecules could potentially have contributed to the enhanced lytic response of HuMax-IL8-treated cells. These results suggest the potential benefit of combining HuMax-IL8 treatment with chemotherapy or immunotherapy for the treatment of TNBC and potentially other tumors driven by an IL-8 autocrine loop.

\section{Discussion}

The chemokine IL-8 has been increasingly implicated in the progression of various types of cancer, including TNBC (31-33). The widespread effects of IL-8 signaling at the tumor site via binding and signaling through the CXCR1 and CXCR2 receptors are twofold: (a) direct effects on tumor cell phenotype that lead 

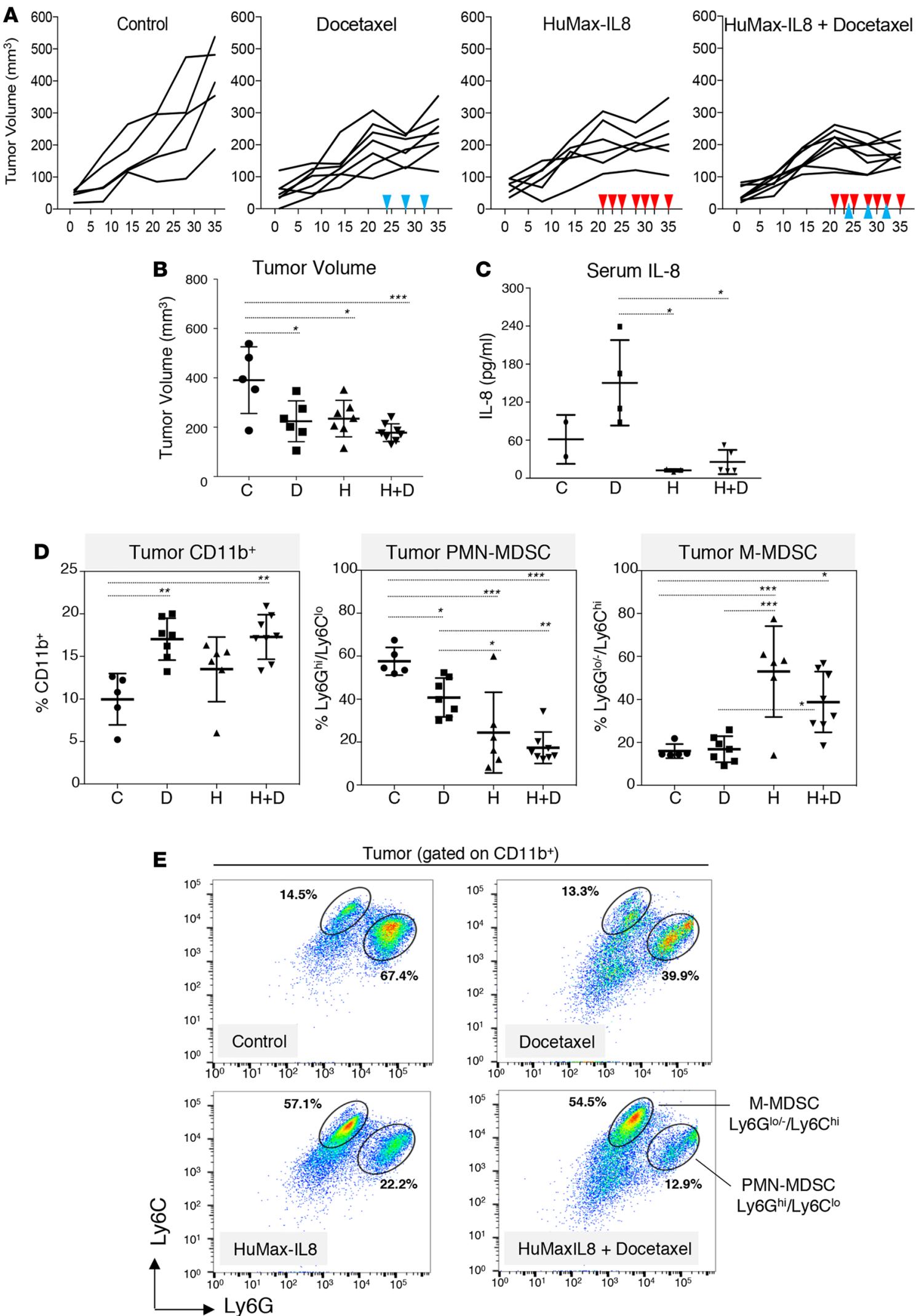

Figure 5. Neutralization of IL-8 reduces tumor growth and recruitment of PMN-MDSCs at the tumor site. Change in tumor volume (A) and tumor volume at the conclusion of the experiment (B) for individual mice treated with control IgC (C), docetaxel (D), HuMax-IL8 (H), or docetaxel and HuMax-IL8 $(\mathrm{H}+\mathrm{D})$. Days of treatment are indicated with blue and red arrowheads for docetaxel and HuMax-IL8, respectively. (C) Human IL-8 detected in the sera collected from individual mice in each treatment group. (D) Quantification of total CD11b+ cell, PMN-MDSC (Ly6G hi/Ly6C ${ }^{10}$ ), and M-MDSC (Ly6G ${ }^{\text {lo/- } / \text { Ly6C }}$ i) populations in individual tumors in each treatment group. (B-D) Data from individual mice (mean \pm SEM); $n=5-8$ mice 
per group. Differences between means were compared using the 1-way ANOVA test with Tukey's multiple comparisons; ${ }^{*} P<0.05 ;{ }^{* *} P<0.01 ;{ }^{* * *} P$ $<0.001$. (E) Representative FACS plots of Ly6C/Ly6C-positive populations on CD11b+ tumor cells for each treatment group. Data are representative of 2 experiments.

to tumor mesenchymalization $(13,14,24)$ and (b) chemotaxis of immune cells, resulting in the recruitment of suppressive MDSC populations (27). Both effects can contribute to therapeutic resistance and cancer progression. Here, various aspects of the biology of IL-8 signaling were explored in in vitro and in vivo models of claudin-low TNBC via the use of an anti-IL-8 targeting monoclonal antibody, HuMax-IL8.

Tumor cell mesenchymalization takes place when epithelial cancer cells lose their polarity, cell-to-cell contacts, and expression of epithelial proteins, including E-cadherin, ZO-1, and cytokeratins, while gaining the expression of proteins normally found in mesenchymal cells, such as fibronectin and vimentin $(34,35)$. Mesenchymalization and the molecular drivers of the process, including the transcription factors Snail, Slug, Twist, and brachyury, have been linked to advanced tumor stage (36-38), presence of metastases (39), and poor prognosis in numerous cancer types (40-42). Additionally, mesenchymalization has been associated with resistance to anticancer therapies, including chemotherapy $(43,44)$, small-molecule-targeted therapies $(45,46)$, and to lysis by immune effector cells $(47-50)$. In the case of breast cancer, this phenomenon has been linked to tumor progression in preclinical models (51) and with patient tumor samples (52, 53). Clinically, the acquisition of mesenchymal markers with or without the loss of epithelial markers has been well-documented in circulating tumor cells (CTCs) from breast cancer patients $(54,55)$. For example, Yu et al. (56) utilized serial monitoring of CTCs during the course of treatment to demonstrate that mesenchymal CTCs are associated with treatment failure and disease progression. Here, we showed the ability of HuMax-IL8 to efficiently reduce mesenchymal features in claudin-low TNBC cell line models both in vitro and in vivo. The modulation of tumor phenotype achieved with HuMax-IL8 is expected to minimize tumor dissemination and metastasis as well as to decrease cancer stemness, including resistance to cytotoxic agents and cytotoxic immune cells. Previous work by others has demonstrated the importance of CXCR1 signaling in the biology of breast cancer stem cells $(15,25)$; blockade of CXCR1, for example, was shown to deplete populations of cancer stem cells in breast cancer cell lines, leading to a substantial reduction of tumor growth and metastasis in vivo. In line with these reports, we have demonstrated the ability of HuMax-IL8 to also reduce the expression of the stemness marker ALDH1A1 in xenografts of MDAMB-231 cells. The capability of HuMax-IL8 to modulate both tumor phenotype and stemness features of claudin-low TNBC cells poses the potential for using this antibody to render tumor cells more amenable to treatment with other therapeutics.

While the immune system retains antitumor capabilities, there are multiple mechanisms of immune suppression that can arise in the disease state to facilitate the progression of cancer $(12,57)$. Among them, tumor recruitment and localization of MDSCs represent one major impediment to effective antitumor immune responses. MDSCs can be subdivided into two groups: M-MDSCs, which are phenotypically and morphologically comparable to monocytes, and PMN-MDSCs, which are comparable to neutrophils $(58,59)$. Once they accumulate at the tumor site, MDSCs exert a suppressive role on immune cells, mainly on T cells, via several immune-suppressive factors, including arginase-1, iNOS, TGF- $\beta$, IL-10, and ROS, among others. Interestingly, different mechanisms appear to be used by the two subgroups of MDSCs, with M-MDSCs mediating immune suppression via expression of high levels of ARG-1 and iNOS and PMN-MDSCs mainly producing high levels of ROS (60). While initially characterized in mouse cancer models (61), expansion of MDSCs is known to take place in many solid tumor types, including breast cancer (26), and high numbers of MDSCs are detected in the peripheral blood of cancer patients, particularly in advanced stages of disease, compared with blood of healthy donors (62). The study of the mechanisms involved in the tumor recruitment of MDSCs has led to the understanding that homing of M-MDSCs is mainly dependent upon the activation of the CCL2/CCR2 axis, while attraction of PMN-MDSCs has been mostly associated with signaling via the CXCR2 receptor, which is in agreement with the predominant expression of CXCR1 and CXCR2 in PMN-MDSCs, as compared with the prevalence of CCR2 expression in M-MDSC populations (63). Accordingly, disruption of CXCR2 signaling has been shown to prevent PMN-MDSC trafficking to murine rhabdomyosarcoma tumors (16).

In the present study, our results demonstrate the ability of HuMax-IL8 to effectively decrease the recruitment of PMN-MDSCs to xenografts of TNBC cells, which resulted in a greater proportion of M-MDSCs within the tumors and a decrease in the ratio of PMN-MDSCs to M-MDSCs. Interestingly, on a per cell basis, 
A Spleen CD11b

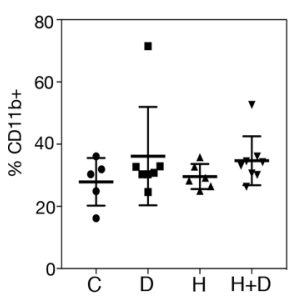

Spleen PMN-MDSC

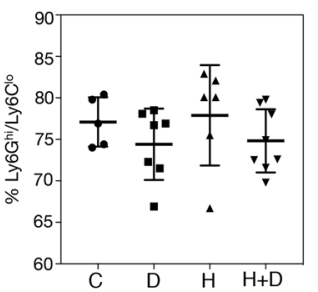

Spleen M-MDSC

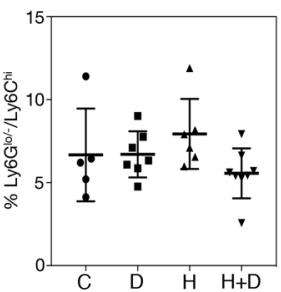

C Bone Marrow CD11b+

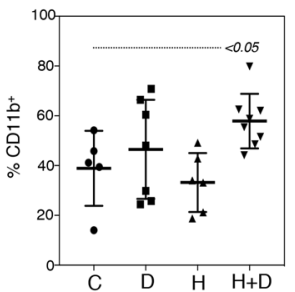

BM PMN-MDSC

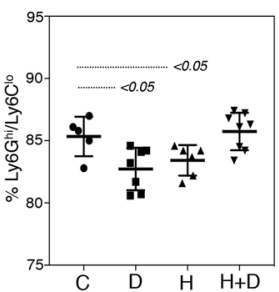

BM M-MDSC

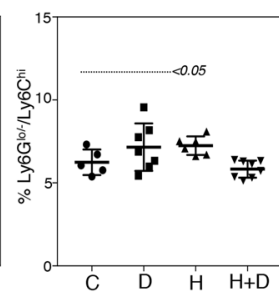

B

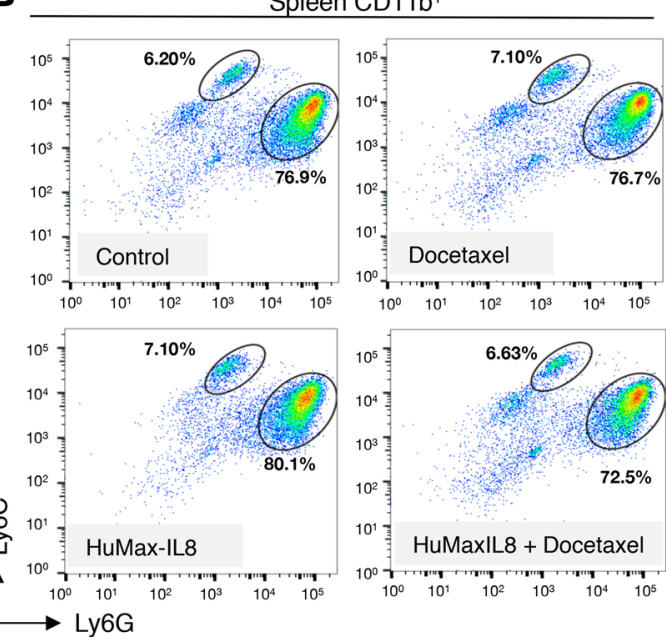

D

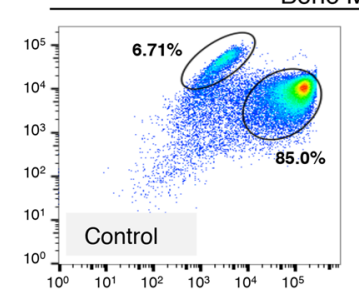

Bone Marrow CD11 $\mathrm{b}^{+}$

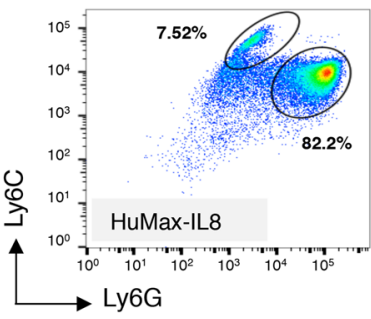

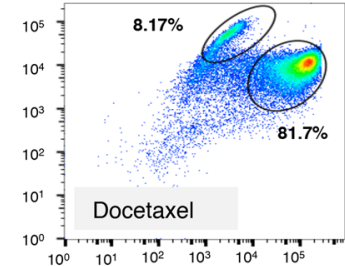

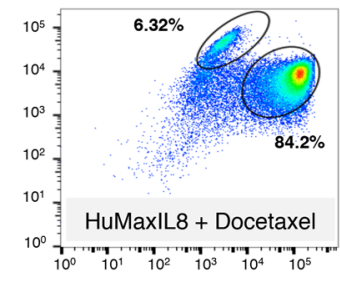

Figure 6. MDSC populations from the spleen and bone marrow are not affected by changes in tumor-secreted human IL-8. Quantification of total CD11 ${ }^{+}$

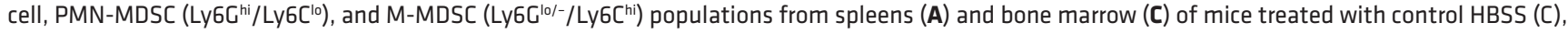
docetaxel (D), HuMax-IL8 (H), and the combination of HuMax-IL8 and docetaxel $(H+D)$. Data represent mean \pm SEM; $n=5-8$ mice per group. Differences between means were compared using the 1-way ANOVA test with Tukey's multiple comparisons. (B and D) Representative FACS plots of Ly6C/Ly6C-positive populations gated on $\mathrm{CD}_{11 b^{+}}$cells for each treatment group in spleen (B) or bone marrow (D) samples. Data are representative of 2 experiments.

M-MDSCs have been shown to exert stronger immune suppression than PMN-MDSCs (64). However, targeted depletion of PMN-MDSCs has been shown to improve the activation and proliferation of intratumoral CD8 ${ }^{+}$ $\mathrm{T}$ cells in an experimental model of pancreatic cancer, even in the presence of an expanded M-MDSC population (65). In addition, depletion of PMN-MDSCs via anti-Ly6G antibody was shown to improve antitumor immunity in a murine lung tumor model, with enhanced frequency and activation of $\mathrm{NK}$ and $\mathrm{T}$ cell effectors in the tumor (66). Changes in the recruitment of PMN-MDSC populations were also assessed in tumors treated with docetaxel, a chemotherapeutic agent that has been previously shown to reduce the proportion of MDSCs in the spleens of tumor-bearing mice (67). Interestingly, the levels of circulating human IL-8 were significantly higher in mice treated with chemotherapy alone, yet this group of mice showed decreased levels of infiltrating PMN-MDSCs at the tumor site. Further studies will need to be conducted to evaluate tumor levels of IL- 8 in response to chemotherapy alone and the potential effect on immune infiltration. Here, we demonstrated that the combination of HuMax-IL8 with docetaxel afforded an even more pronounced decrease in PMN-MDSCs than that observed with either treatment alone. These results highlight the possible value that HuMax-IL8 treatment could add to current therapies, wherein blockade of IL- 8 could aid by reverting mesenchymalization of tumor cells, while simultaneously hindering the trafficking of MDSCs to the tumor site.

Interestingly, the role of MDSCs in promoting cancer progression is not limited to their ability to inhibit T cells via the release of suppressive soluble factors (68-70), but it also involves their ability to promote both tumor mesenchymalization $(71,72)$ and resistance to immune-mediated killing $(73,74)$. In TNBC in particular, Suarez-Carmona et al. (75) studied the interaction between tumor mesenchymal features and inflammatory cytokine signaling and demonstrated a positive association between mesenchymalization-induced IL-8 secretion and the recruitment of granulocytic MDSCs. Thus, the effect of HuMax-IL8 in reducing tumor-infiltrating MDSCs could contribute to reducing mesenchymal features in the cancer cells, and, in turn, the loss of mesenchymalization could reduce secretion of IL- 8 by the tumor cells, further decreasing 
A

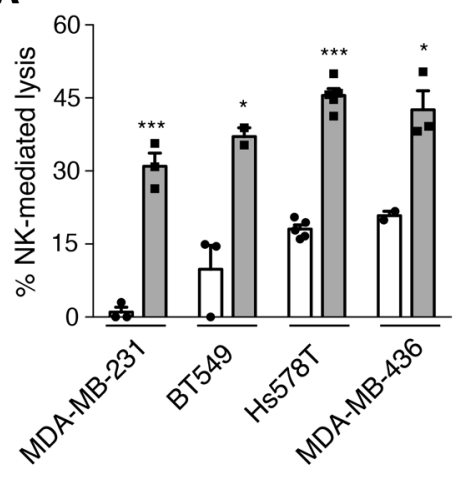

B

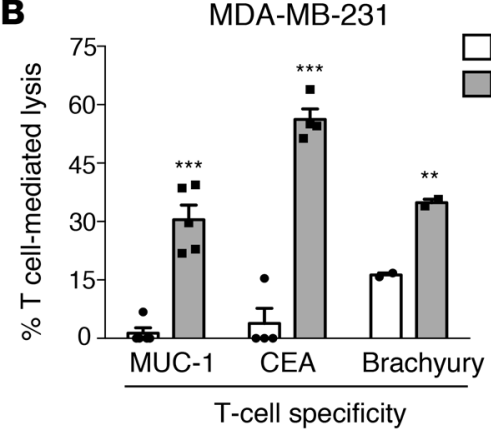

C MDA-MB-231

Hs578T Control $\lg G$ HuMax-IL8

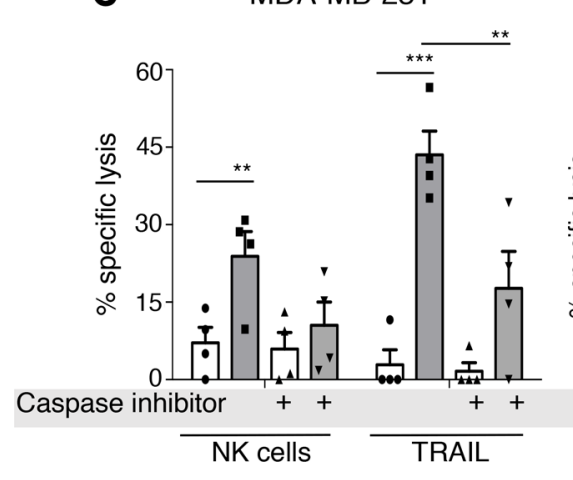

D

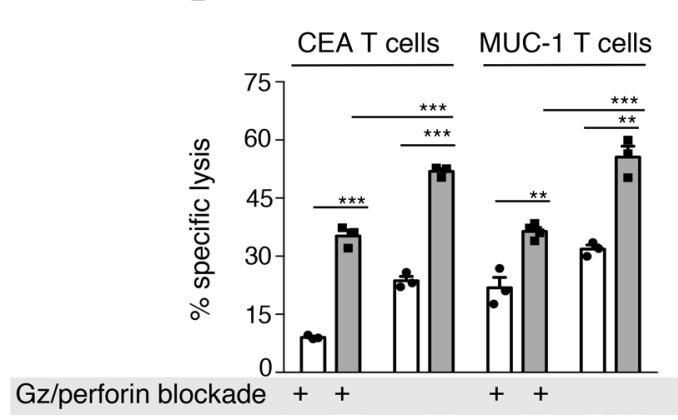

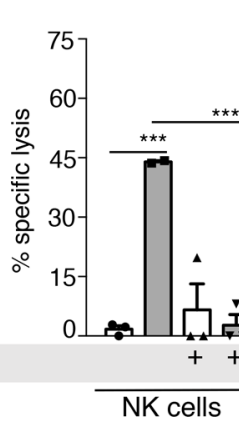

cells

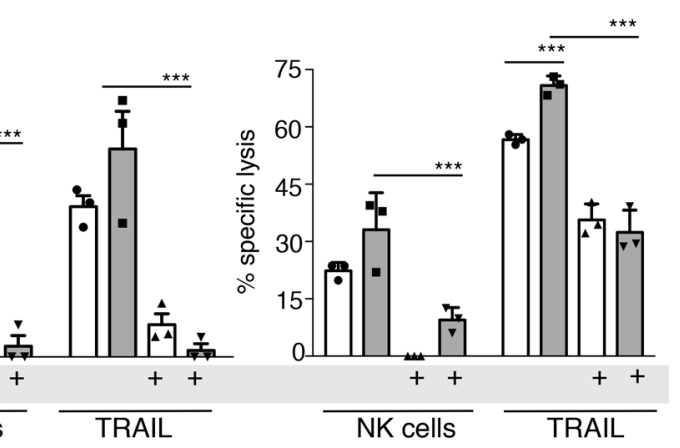
blood of 2-3 healthy donors, following tumor cell treatment with HuMax-IL8 versus control IgC for 3 days. Data represent mean (bars) + SEM (error bars), $n=3$ (dots); difference between means was assessed with 2-tailed $t$ test for each TNBC cell line. Data are representative of 3 experiments. (B) Susceptibility of MDA-MB-231 cells treated with control HuMax-IL8 versus control IgG to lysis by MUC-1-, CEA-, and brachyury-specific CD8+ T cells. Data represent mean (bars) + SEM (error bars), $n=3-4$ (dots); difference between means was assessed with 2-tailed $t$ test for each antigen-specific T cell line. Data are representative of 2 experiments. (C) NK- and TRAIL-mediated lysis of MDA-MB-231, Hs578T, and MDA-MB-436 cells that were untreated or pretreated with the pan-caspase inhibitor Z-VAD-FMK prior to the cytotoxic assay. Data represent mean (bars) \pm SEM (error bars), $n=3-4$ (dots); differences between means were compared with 1-way ANOVA test with Tukey's multiple comparisons. Data are from 1 experiment. (D) CEA- and MUC-1-specific CD8+ T cells were left untreated or pretreated with CMA for blockade of granzyme/perforin activity and utilized for lysis with MDA-MB-231 and BT549 target cells treated with HuMax-IL8 versus control IgG. Data represent mean (bars) \pm SEM (error bars), $n=3$ (dots); differences between means were compared with 1 -way ANOVA test with Tukey's multiple comparisons. Data are representative of 2 experiments. ${ }^{*} P<0.05 ;{ }^{*} P<0.01 ;{ }^{* *} P<0.001$.

the recruitment of MDSCs to the tumor site. Furthermore, as described above, the reversion of mesenchymal features could render cancer cells more responsive to immune-mediated lysis $(76,77)$, and the reduction of MDSC recruitment to the tumor site has been reported to enhance the response to vaccination (78).

In agreement with previous reports demonstrating a role for tumor mesenchymalization in tumor resistance to immune-mediated cytotoxicity $(48,76,79)$, the present work showed the ability of HuMaxIL8 to revert tumor phenotype, while significantly enhancing the susceptibility of claudin-low TNBC cells to lysis by immune effector NK and cytotoxic $\mathrm{CD}^{+} \mathrm{T}$ cells and by TRAIL. Regarding the mechanism 
involved in this enhanced lysis, we demonstrated that blockade of IL-8 signaling in mesenchymal carcinoma cells leads to a significant increase in the tumor cells' susceptibility to caspase-mediated cell lysis, while only partially improving the perforin/granzyme lytic pathway. This is in agreement with the previous observation that carcinoma cells with mesenchymal features are resistant to caspase-dependent apoptosis, compared with their epithelial counterparts (48). We thus hypothesize that the reversion of phenotype achieved via blockade of IL- 8 signaling could alleviate the apoptotic defects that are characteristic of mesenchymalized tumor cells, leading to a subsequent improvement of cytotoxicity. The improvement of immune-mediated lysis following HuMax-IL8 treatment was also found to be independent of changes in the expression of MHC, costimulatory, or inhibitory molecules. However, HuMax-IL8 treatment also increased the expression of various molecules involved in antigen processing and presentation, which, together with an enhancement in TRAIL receptors, could potentially contribute to the enhanced lytic response of HuMax-IL8-treated cells.

While numerous reports have implicated IL- 8 in various aspects of cancer progression, here the ability of HuMax-IL8 to alter the interactions between claudin-low TNBC cells and the surrounding immune infiltrate was explored for the first time to our knowledge. HuMax-IL8 was evaluated in the clinic, including in a recently completed phase Ib clinical trial (NCT02536469) conducted in patients with metastatic or unresectable, locally advanced malignant solid tumors. Our demonstration of the ability of this antibody to markedly reduce tumor mesenchymalization, significantly reduce MDSC recruitment to the tumor site, and enhance the susceptibility of claudin-low TNBC cells to immune-mediated lysis validates HuMax-IL8 as a potential agent for combination therapy with chemotherapy or immunotherapy for the treatment of claudin-low TNBC and other tumors in which IL-8 contributes to disease progression. Moreover, based on work conducted by others (17) demonstrating that blockade of IL- 8 could significantly reduce the proliferation of basal-like TNBC cells, neutralization of IL-8 with HuMax-IL8 could also be applied to the basal-like subtype of TNBC, thus expanding the potential applicability of the approach to the majority of TNBC tumor types.

\section{Methods}

Tumor cell lines and tissue culture. The MDA-MB-231, MDA-MB-436, Hs578T, BT549, HCC1143, ZR-75-1, and MCF7 breast cancer cell lines were obtained from ATCC and propagated as recommended. SUM149PT cells were obtained from Asterand Bioscience. Cells were used for experiments within 12 passages from the time of purchase or were authenticated (SUM149PT, ZR-75-1, MCF7) by short tandem repeat analysis (Bio-Synthesis Inc.) and used within 5 passages of verification.

Anti-IL-8 monoclonal antibody. HuMax-IL8 is a fully human, high-affinity monoclonal antibody directed against IL-8 (formerly known as HuMab 10F8), which, upon binding to IL-8, inhibits its binding to the IL-8 receptors CXCR1 and CXCR2. HuMax-IL8 was obtained under the terms of a material transfer agreement between Cormorant Pharmaceuticals and the National Cancer Institute, NIH.

ELISA and cytokine array. An ELISA assay (RayBiotech) was used for detection of human IL-8 in culture supernatants from the various cell lines grown for 24 hours in RPM1-1640 medium (Corning) supplemented with $10 \%$ fetal bovine serum (FBS, Gemini) at $1 \times 10^{6}$ cells per $1.5 \mathrm{ml}$. For experiments involving neutralization of IL-8 with HuMax-IL8, TNBC cells were cultured at $1 \times 10^{6}$ cells per $1.5 \mathrm{ml}$ in medium supplemented with $2 \%$ FBS in the presence of $25 \mu \mathrm{g} / \mathrm{ml}$ of a purified human IgG (Sigma-Aldrich) or HuMax-IL8 for 3 days. The ELISA assay was confirmed not to react with IL-8 bound to HuMax-IL8. At the time of supernatant collection, cells were harvested and counted, and the results were adjusted for final cell count.

Detection of multiple cytokines, chemokines, and growth factors was conducted in culture supernatants of MDA-MB-231 and MDA-MB-436 cells grown as indicated above, utilizing a Human Cytokine Antibody Array (C-series, Ray Biotech), following the manufacturer's recommendations.

Cell viability. For cell viability assays, each cell line was plated at $1 \times 10^{6}$ cells per $1.5 \mathrm{ml}$ in the presence of $25 \mu \mathrm{g} / \mathrm{ml}$ human IgG (Sigma-Aldrich) or HuMax-IL8 in media supplemented with 2\% FBS for a total of 4 days. Cells were harvested and counted with trypan blue to assess the number of viable cells.

Immunofluorescence. Tumor cells were grown in flat-bottom 96 -well plates, fixed with $4 \%$ formaldehyde, permeabilized with $0.2 \%$ Triton-X, and, subsequently, blocked with $1 \times$ PBS containing $10 \%$ goat serum and $1 \%$ BSA. Primary antibodies directed against E-cadherin (36E), fibronectin (10F, BD Biosciences), CXCR1 (MM0221-7D22, Abcam), and CXCR2 (20634-1-AP, ProteinTech) were added overnight at $4^{\circ} \mathrm{C}$ in $1 \times$ PBS 
containing 1\% BSA, followed by addition of Alexa Fluor 488-conjugated secondary antibody (A11029, A11008, ThermoFisher Scientific) for 1 hour at room temperature. DAPI was added at 1:1,000 dilution in $1 \times$ PBS for 5 minutes, and rhodamine-conjugated phalloidin (Life Technologies) was added at $2 \mu 1 /$ well in $1 \times$ PBS for 20 minutes. Images were captured utilizing a Leica Fluorescent microscope (magnification $\times 20$ unless indicated otherwise). Fluorescence was quantified utilizing ImageJ (NIH) binary pixel intensity analysis, and the ratio of E-cadherin over fibronectin was used as a measure of mesenchymalization.

Tumor studies in vivo. To establish orthotopic tumors in the mammary fat pad, 6-week-old female C.B-17 SCID mice (Taconic) were inoculated with $4 \times 10^{6}$ cells in $50 \mu 1$ HBSS admixed with Matrigel 50\% (v/v, Corning). All mice were housed and maintained in microisolator cages under specific pathogen-free conditions and in accordance with the Association for Assessment and Accreditation of Laboratory Animal Care guidelines. For experiments involving HuMax-IL8 monotherapy, mice were randomized when tumors became palpable (day 1) and received 2 i.p. injections of $200 \mu \mathrm{g}$ of a control human IgG (Sigma-Aldrich) or HuMax-IL8 on days 1 and 3; tumors were collected on day 6 and processed as indicated below. For combination studies, control HBSS (100 $\mu$, Corning), $10 \mathrm{mg} /$ $\mathrm{kg}$ docetaxel (Winthrop US), $200 \mu \mathrm{g}$ HuMax-IL8, or a combination of docetaxel and HuMax-IL8 were given i.p. when tumors became palpable (day 1). Mice were injected with HuMax-IL8 on days 1, 3, 5, $8,10,12$, and 15; docetaxel was given on days 4,8 , and 12; tumors were collected on day 17. Half of the tumor tissue was formalin-fixed and processed for immunohistochemical evaluation using primary antibodies against fibronectin (GTX112794, GeneTex), vimentin (SP20, ThermoFisher Scientific), ZO-1 (GTX108613, GeneTex), E-cadherin (EP700Y, ThermoFisher Scientific), and ALDH1A1 (NBP2-15336, Novus Biologicals), as indicated below. The remaining tumor tissue was dissociated with a mouse tumor dissociation kit (Miltenyi Biotec). Spleen and bone marrow samples were also collected and dissociated manually. Single-cell suspensions from tumor, spleen, and bone marrow were incubated with ACK lysis buffer (Quality Biological) for 5 minutes at $4^{\circ} \mathrm{C}$, washed, and used for flow cytometry utilizing the following antibodies from BD Biosciences: anti-mouse CD11b (M1/70), Ly6G (1A8), Ly6C (AL-21), and F4/80 (clone BM8, ThermoFisher Scientific). Blood samples were also collected through cardiac puncture for serum separation. Tumor-derived, circulating human IL-8 was measured in serum samples via ELISA assay (RayBioTech).

Immunohistochemistry. Formalin-fixed tumor tissue sections were heated at $65^{\circ} \mathrm{C}$ for 45 minutes, deparaffinized with xylene, and then hydrated through a graded series of ethanol concentrations. After antigen retrieval was performed at $95^{\circ} \mathrm{C}$ for 20 minutes in a $\mathrm{pH}-9$ solution (Dako), tumor sections were permeabilized with PBS containing $0.1 \%$ Triton X-100. Endogenous peroxidase activity was blocked using a $3 \%$ hydrogen peroxide solution. Sections were subsequently blocked with $100 \%$ horse serum for 30 minutes and then incubated in primary antibody for 90 minutes at room temperature, followed by peroxidase-conjugated secondary antibodies (Vector Laboratories) for 30 minutes. Immunostaining was developed with DAB peroxidase substrate (Vector Laboratories); sections were counterstained with Mayer's hematoxylin, dehydrated, and mounted. Images were captured using a Leica microscope. Slides were digitally scanned with an Aperio ScanScope scanning system (Aperio Technologies Inc.) and analyzed by using the Aperio ImageScope Viewer software. An entire tissue section was selected and necrotic regions were excluded with negative gate. The positive pixel count algorithm was used to measure the intensity of each marker (brown signal). Weak and strong positive staining was recorded for the whole tumor section, and the percentage weak and strong positive pixels was calculated relative to the total number of pixels in the section.

Flow cytometry. Cells from tumor, spleen, and bone marrow were prepared as described above. Cells were stained utilizing a multicolor method to assess coexpression of various markers on live immune cells. For MDSC populations, the single-cell population was gated (FSC-A, FSC-H), followed by gating on live cells by using a LIVE/DEAD Fixable Aqua Dead Cell Stain Kit, $405 \mathrm{~nm}$ excitation (Life Technologies). After gating on the $\mathrm{CD} 11 \mathrm{~b}^{+}$population, PMN-MDSC fractions were defined as $\mathrm{Ly} 6 \mathrm{G}^{\mathrm{hi}} / \mathrm{Ly} 6 \mathrm{C}^{\mathrm{lo} /-}$, and M-MDSC fractions as Ly6C $\mathrm{Ci}^{\mathrm{hi}} / \mathrm{Ly} 6 \mathrm{G}^{\mathrm{lo} /-}$.

Chemotaxis assay. $\mathrm{CD} 11 \mathrm{~b}^{+}$cells were isolated using magnetic beads (Miltenyi Biotec) from spleens from 2-3 wild-type BALB/c mice. Media containing IgG isotype control (25 $\mu \mathrm{g} / \mathrm{ml})$ (Sigma-Aldrich), recombinant human IL-8 (100 ng/ml, Peprotech), HuMax-IL8 $(25 \mu \mathrm{g} / \mathrm{ml})$, or a combination were added to the lower portion of Boyden chambers with $5-\mu \mathrm{m}$ filters (NeuroProbe Inc.). CD11 b cells were placed on the top chambers at $1 \times 10^{5}$ cells and incubated overnight. Cells were counted in the lower portion of the chamber. Each condition was assayed in 3-4 replicate chambers, as indicated. 
Immune effector cells. NK cells were isolated from healthy donor peripheral blood mononuclear cells by using a magnetic NK Cell Isolation Kit (Miltenyi Biotech) and were cultured in RPMI media containing $10 \%$ FBS. Antigen-specific, HLA-A ${ }_{02}$ - and HLA-A ${ }_{24}$-restricted cytotoxic T lymphocytes directed against an epitope of brachyury (WLLPGTSTV) or mucin-1 (MUC-1, KYHPMSEYAL), respectively, were generated and expanded as previously described from the blood of cancer patients (80-82).

Cytotoxicity assay. Target cells were incubated with $25 \mu \mathrm{g} / \mathrm{ml}$ of control IgG or HuMax-IL8 for 3-6 days in RPMI media containing 2\% FBS. On the day of the assay, cells were harvested, washed, and labeled with $10 \mu \mathrm{M}$ calcein-AM (Invitrogen) for 15 minutes at $37^{\circ} \mathrm{C}$ and subsequently plated at $2 \times 10^{3}$ to $3 \times 10^{3}$ cells per well in 96-well flat-bottom culture plates. Target cells were cocultured with effector

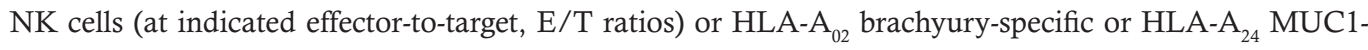
specific $\mathrm{CD}^{+} \mathrm{T}$ cells (at 35:1 E/T ratio). TRAIL was used at 250-500 ng/ml, where indicated. Following overnight culture at $37^{\circ} \mathrm{C}$, propidium iodide was added at $2 \mu \mathrm{g} / \mathrm{ml}$; viable cells were counted with a Celigo Image Cytometer (Nexcellom Bioscience). Spontaneous release was determined by incubating target cells with medium alone; all determinations were done in 6 replicates. Specific lysis was calculated as follows: specific lysis $(\%)=100-[$ (observed viability/spontaneous viability $) \times 100]$. For blockade experiments, a pan-caspase inhibitor (Z-VAD-FMK, CalBiochem) was added to the target cells at $50 \mu \mathrm{M} / \mathrm{ml}$ for 1 hour at $37^{\circ} \mathrm{C}$, prior to the assay. For blockade of granzyme/perforin, effector cells were incubated with CMA (Sigma-Aldrich) at $200 \mathrm{nM} / \mathrm{ml}$ for 2 hours at $37^{\circ} \mathrm{C}$ prior to the assay.

Statistics. Data were analyzed using GraphPad Prism (GraphPad Software) utilizing the 2-tailed, unpaired $t$ test for triplicate or more measurements, with $P<0.05$ considered as statistically significant. For multiple comparisons, 1-way ANOVA, with Tukey's multiple comparisons test was used. Data points in graphs represent mean \pm SEM.

Study approval. All experimental murine in vivo studies were carried out under the approval of the NIH Intramural Animal Care and Use Committee and in accordance with the Association for Assessment and Accreditation of Laboratory Animal Care guidelines. Peripheral blood mononuclear cells from healthy donors and cancer patients were obtained under the approval of the appropriate institutional review board of the NIH and with informed consent.

\section{Author contributions}

$\mathrm{CD}$ designed research studies, conducted experiments, acquired and analyzed data, and wrote the manuscript. JMD and KKM conducted experiments and acquired and analyzed data. CP designed and supervised studies, analyzed data, and wrote the manuscript.

\section{Acknowledgments}

The authors gratefully acknowledge financial support from the Intramural Research Program of the Center for Cancer Research, National Cancer Institute, NIH. The authors thank Debra Weingarten for assistance with the preparation of the manuscript.

Address correspondence to: Claudia Palena, Laboratory of Tumor Immunology and Biology, Center for Cancer Research, National Cancer Institute, National Institutes of Health, 10 Center Drive, Room 8B14, MSC 1750, Bethesda, Maryland 20892, USA. Phone: 301.496.1528; Email: palenac@mail.nih.gov.

1. Miller KD, et al. Cancer treatment and survivorship statistics, 2016. CA Cancer J Clin. 2016;66(4):271-289.

2. Metzger-Filho O, et al. Dissecting the heterogeneity of triple-negative breast cancer. J Clin Oncol. 2012;30(15):1879-1887.

3. Dent R, et al. Triple-negative breast cancer: clinical features and patterns of recurrence. Clin Cancer Res. 2007;13(15 Pt 1):4429-4434.

4. Prat A, et al. Phenotypic and molecular characterization of the claudin-low intrinsic subtype of breast cancer. Breast Cancer Res. 2010;12(5):R68.

5. Prat A, Perou CM. Deconstructing the molecular portraits of breast cancer. Mol Oncol. 2011;5(1):5-23.

6. Creighton CJ, et al. Residual breast cancers after conventional therapy display mesenchymal as well as tumor-initiating features Proc Natl Acad Sci USA. 2009;106(33):13820-13825.

7. Cleator S, Heller W, Coombes RC. Triple-negative breast cancer: therapeutic options. Lancet Oncol. 2007;8(3):235-244.

8. Baggiolini M, Walz A, Kunkel SL. Neutrophil-activating peptide-1/interleukin 8, a novel cytokine that activates neutrophils. J Clin Invest. 1989;84(4):1045-1049.

9. Yoshimura T, et al. Purification of a human monocyte-derived neutrophil chemotactic factor that has peptide sequence similarity to other host defense cytokines. Proc Natl Acad Sci USA. 1987;84(24):9233-9237.

10. David JM, Dominguez C, Hamilton DH, Palena C. The IL-8/IL-8R axis: a double agent in tumor immune resistance. Vaccines 
(Basel). 2016;4(3):E22.

11. Benoy IH, et al. Increased serum interleukin- 8 in patients with early and metastatic breast cancer correlates with early dissemination and survival. Clin Cancer Res. 2004;10(21):7157-7162.

12. Alfaro C, et al. Tumor-produced interleukin-8 attracts human myeloid-derived suppressor cells and elicits extrusion of neutrophil extracellular traps (NETs). Clin Cancer Res. 2016;22(15):3924-3936.

13. Fernando RI, Castillo MD, Litzinger M, Hamilton DH, Palena C. IL-8 signaling plays a critical role in the epithelial-mesenchymal transition of human carcinoma cells. Cancer Res. 2011;71(15):5296-5306.

14. Palena C, Hamilton DH, Fernando RI. Influence of IL- 8 on the epithelial-mesenchymal transition and the tumor microenvironment. Future Oncol. 2012;8(6):713-722.

15. Ginestier C, et al. CXCR1 blockade selectively targets human breast cancer stem cells in vitro and in xenografts. J Clin Invest. 2010;120(2):485-497.

16. Highfill SL, et al. Disruption of CXCR2-mediated MDSC tumor trafficking enhances anti-PD1 efficacy. Sci Transl Med. 2014;6(237):237ra67.

17. Zheng ZY, et al. Wild-Type N-Ras, Overexpressed in basal-like breast cancer, promotes tumor formation by inducing il-8 secretion via JAK2 activation. Cell Rep. 2015;12(3):511-524.

18. Schott AF, et al. Phase $\mathrm{Ib}$ pilot study to evaluate reparixin in combination with weekly paclitaxel in patients with HER2-negative metastatic breast cancer. Clin Cancer Res. 2017;23(18):5358-5365.

19. Skov L, et al. IL-8 as antibody therapeutic target in inflammatory diseases: reduction of clinical activity in palmoplantar pustulosis. J Immunol. 2008;181(1):669-679.

20. Bangsgaard N, et al. Neutralization of IL-8 prevents the induction of dermatologic adverse events associated with the inhibition of epidermal growth factor receptor. PLoS ONE. 2012;7(6):e39706.

21. Prat A, et al. Characterization of cell lines derived from breast cancers and normal mammary tissues for the study of the intrinsic molecular subtypes. Breast Cancer Res Treat. 2013;142(2):237-255.

22. Bates RC, DeLeo MJ, Mercurio AM. The epithelial-mesenchymal transition of colon carcinoma involves expression of IL-8 and CXCR-1-mediated chemotaxis. Exp Cell Res. 2004;299(2):315-324.

23. Visciano C, et al. Mast cells induce epithelial-to-mesenchymal transition and stem cell features in human thyroid cancer cells through an IL-8-Akt-Slug pathway. Oncogene. 2015;34(40):5175-5186.

24. Fernando RI, Hamilton DH, Dominguez C, David JM, McCampbell KK, Palena C. IL-8 signaling is involved in resistance of lung carcinoma cells to erlotinib. Oncotarget. 2016;7(27):42031-42044.

25. Charafe-Jauffret $\mathrm{E}$, et al. Breast cancer cell lines contain functional cancer stem cells with metastatic capacity and a distinct molecular signature. Cancer Res. 2009;69(4):1302-1313.

26. Danilin S, Merkel AR, Johnson JR, Johnson RW, Edwards JR, Sterling JA. Myeloid-derived suppressor cells expand during breast cancer progression and promote tumor-induced bone destruction. Oncoimmunology. 2012;1(9):1484-1494

27. Katoh H, Wang D, Daikoku T, Sun H, Dey SK, Dubois RN. CXCR2-expressing myeloid-derived suppressor cells are essential to promote colitis-associated tumorigenesis. Cancer Cell. 2013;24(5):631-644

28. Asfaha S, et al. Mice that express human interleukin- 8 have increased mobilization of immature myeloid cells, which exacerbates inflammation and accelerates colon carcinogenesis. Gastroenterology. 2013;144(1):155-166.

29. Collins TS, Lee LF, Ting JP. Paclitaxel up-regulates interleukin-8 synthesis in human lung carcinoma through an NF-kappaBand AP-1-dependent mechanism. Cancer Immunol Immunother. 2000;49(2):78-84.

30. De Larco JE, Wuertz BR, Manivel JC, Furcht LT. Progression and enhancement of metastatic potential after exposure of tumor cells to chemotherapeutic agents. Cancer Res. 2001;61(7):2857-2861.

31. Shao $\mathrm{N}$, et al. Interleukin- 8 upregulates integrin $\beta 3$ expression and promotes estrogen receptor-negative breast cancer cell invasion by activating the PI3K/Akt/NF-kB pathway. Cancer Lett. 2015;364(2):165-172.

32. Rody A, et al. A clinically relevant gene signature in triple negative and basal-like breast cancer. Breast Cancer Res. 2011;13(5):R97.

33. Kim S, Lee J, Jeon M, Lee JE, Nam SJ. MEK-dependent IL-8 induction regulates the invasiveness of triple-negative breast cancer cells. Tumour Biol. 2016;37(4):4991-4999.

34. Thiery JP, Acloque H, Huang RY, Nieto MA. Epithelial-mesenchymal transitions in development and disease. Cell. 2009;139(5):871-890.

35. Kalluri R. EMT: when epithelial cells decide to become mesenchymal-like cells. J Clin Invest. 2009;119(6):1417-1419.

36. Hotz B, Arndt M, Dullat S, Bhargava S, Buhr HJ, Hotz HG. Epithelial to mesenchymal transition: expression of the regulators snail, slug, and twist in pancreatic cancer. Clin Cancer Res. 2007;13(16):4769-4776.

37. Cheng GZ, Chan J, Wang Q, Zhang W, Sun CD, Wang LH. Twist transcriptionally up-regulates AKT2 in breast cancer cells leading to increased migration, invasion, and resistance to paclitaxel. Cancer Res. 2007;67(5):1979-1987.

38. Jin H, et al. Snail is critical for tumor growth and metastasis of ovarian carcinoma. Int J Cancer. 2010;126(9):2102-2111.

39. Zhao D, et al. Cytoplasmic p27 promotes epithelial-mesenchymal transition and tumor metastasis via STAT3-mediated Twist1 upregulation. Oncogene. 2015;34(43):5447-5459.

40. Roselli M, et al. Brachyury, a driver of the epithelial-mesenchymal transition, is overexpressed in human lung tumors: an opportunity for novel interventions against lung cancer. Clin Cancer Res. 2012;18(14):3868-3879.

41. Palena C, et al. Overexpression of the EMT driver brachyury in breast carcinomas: association with poor prognosis. J Natl Cancer Inst. 2014;106(5):dju054.

42. Spaderna S, et al. A transient, EMT-linked loss of basement membranes indicates metastasis and poor survival in colorectal cancer. Gastroenterology. 2006;131(3):830-840.

43. Deng J, et al. Targeting epithelial-mesenchymal transition and cancer stem cells for chemoresistant ovarian cancer. Oncotarget. 2016;7(34):55771-55788.

44. Lim S, Becker A, Zimmer A, Lu J, Buettner R, Kirfel J. SNAI1-mediated epithelial-mesenchymal transition confers chemoresistance and cellular plasticity by regulating genes involved in cell death and stem cell maintenance. PLoS One. 2013;8(6):e66558.

45. Arumugam T, et al. Epithelial to mesenchymal transition contributes to drug resistance in pancreatic cancer. Cancer Res. 
2009;69(14):5820-5828.

46. Suda K, et al. Epithelial to mesenchymal transition in an epidermal growth factor receptor-mutant lung cancer cell line with acquired resistance to erlotinib. J Thorac Oncol. 2011;6(7):1152-1161.

47. Kudo-Saito C, Shirako H, Takeuchi T, Kawakami Y. Cancer metastasis is accelerated through immunosuppression during Snailinduced EMT of cancer cells. Cancer Cell. 2009;15(3):195-206.

48. Hamilton DH, Huang B, Fernando RI, Tsang KY, Palena C. WEE1 inhibition alleviates resistance to immune attack of tumor cells undergoing epithelial-mesenchymal transition. Cancer Res. 2014;74(9):2510-2519.

49. Hamilton DH, et al. Targeting estrogen receptor signaling with fulvestrant enhances immune and chemotherapy-mediated cytotoxicity of human lung cancer. Clin Cancer Res. 2016;22(24):6204-6216.

50. David JM, Hamilton DH, Palena C. MUC1 upregulation promotes immune resistance in tumor cells undergoing brachyurymediated epithelial-mesenchymal transition. Oncoimmunology. 2016;5(4):e1117738.

51. Bonnomet A, et al. A dynamic in vivo model of epithelial-to-mesenchymal transitions in circulating tumor cells and metastases of breast cancer. Oncogene. 2012;31(33):3741-3753.

52. Sarrió D, Rodriguez-Pinilla SM, Hardisson D, Cano A, Moreno-Bueno G, Palacios J. Epithelial-mesenchymal transition in breast cancer relates to the basal-like phenotype. Cancer Res. 2008;68(4):989-997.

53. Sethi S, et al. Molecular markers of epithelial-to-mesenchymal transition are associated with tumor aggressiveness in breast carcinoma. Transl Oncol. 2011;4(4):222-226.

54. Aktas B, Tewes M, Fehm T, Hauch S, Kimmig R, Kasimir-Bauer S. Stem cell and epithelial-mesenchymal transition markers are frequently overexpressed in circulating tumor cells of metastatic breast cancer patients. Breast Cancer Res. 2009;11(4):R46.

55. Kallergi G, Papadaki MA, Politaki E, Mavroudis D, Georgoulias V, Agelaki S. Epithelial to mesenchymal transition markers expressed in circulating tumour cells of early and metastatic breast cancer patients. Breast Cancer Res. 2011;13(3):R59.

56. Yu M, et al. Circulating breast tumor cells exhibit dynamic changes in epithelial and mesenchymal composition. Science. 2013;339(6119):580-584.

57. Whiteside TL, Demaria S, Rodriguez-Ruiz ME, Zarour HM, Melero I. Emerging opportunities and challenges in cancer immunotherapy. Clin Cancer Res. 2016;22(8):1845-1855.

58. Bronte V, et al. Recommendations for myeloid-derived suppressor cell nomenclature and characterization standards. Nat Commun. 2016;7:12150.

59. Gabrilovich DI. Myeloid-derived suppressor cells. Cancer Immunol Res. 2017;5(1):3-8.

60. Gabrilovich DI, Ostrand-Rosenberg S, Bronte V. Coordinated regulation of myeloid cells by tumours. Nat Rev Immunol. 2012;12(4):253-268

61. Melani C, Sangaletti S, Barazzetta FM, Werb Z, Colombo MP. Amino-biphosphonate-mediated MMP-9 inhibition breaks the tumor-bone marrow axis responsible for myeloid-derived suppressor cell expansion and macrophage infiltration in tumor stroma. Cancer Res. 2007;67(23):11438-11446.

62. Diaz-Montero CM, Salem ML, Nishimura MI, Garrett-Mayer E, Cole DJ, Montero AJ. Increased circulating myeloid-derived suppressor cells correlate with clinical cancer stage, metastatic tumor burden, and doxorubicin-cyclophosphamide chemotherapy. Cancer Immunol Immunother. 2009;58(1):49-59.

63. Zhao W, et al. Subsets of myeloid-derived suppressor cells in hepatocellular carcinoma express chemokines and chemokine receptors differentially. Int Immunopharmacol. 2015;26(2):314-321.

64. Dolcetti L, et al. Hierarchy of immunosuppressive strength among myeloid-derived suppressor cell subsets is determined by GM-CSF. Eur J Immunol. 2010;40(1):22-35.

65. Stromnes IM, et al. Targeted depletion of an MDSC subset unmasks pancreatic ductal adenocarcinoma to adaptive immunity Gut. 2014;63(11):1769-1781.

66. Srivastava MK, et al. Myeloid suppressor cell depletion augments antitumor activity in lung cancer. PLoS ONE. 2012;7(7):e40677.

67. Kodumudi KN, Woan K, Gilvary DL, Sahakian E, Wei S, Djeu JY. A novel chemoimmunomodulating property of docetaxel: suppression of myeloid-derived suppressor cells in tumor bearers. Clin Cancer Res. 2010;16(18):4583-4594.

68. Marvel D, Gabrilovich DI. Myeloid-derived suppressor cells in the tumor microenvironment: expect the unexpected. J Clin Invest. 2015;125(9):3356-3364.

69. Parker KH, Beury DW, Ostrand-Rosenberg S. Myeloid-derived suppressor cells: critical cells driving immune suppression in the tumor microenvironment. Adv Cancer Res. 2015;128:95-139.

70. Gabrilovich DI, Nagaraj S. Myeloid-derived suppressor cells as regulators of the immune system. Nat Rev Immunol. 2009;9(3):162-174.

71. Sangaletti S, et al. Mesenchymal transition of high-grade breast carcinomas depends on extracellular matrix control of myeloid suppressor cell activity. Cell Rep. 2016;17(1):233-248.

72. Toh B, et al. Mesenchymal transition and dissemination of cancer cells is driven by myeloid-derived suppressor cells infiltrating the primary tumor. PLoS Biol. 2011;9(9):e1001162.

73. Lu T, et al. Tumor-infiltrating myeloid cells induce tumor cell resistance to cytotoxic T cells in mice. J Clin Invest. 2011;121(10):4015-4029.

74. Lindau D, Gielen P, Kroesen M, Wesseling P, Adema GJ. The immunosuppressive tumour network: myeloid-derived suppressor cells, regulatory T cells and natural killer T cells. Immunology. 2013;138(2):105-115.

75. Suarez-Carmona M, et al. Soluble factors regulated by epithelial-mesenchymal transition mediate tumour angiogenesis and myeloid cell recruitment. J Pathol. 2015;236(4):491-504.

76. Dominguez C, Tsang KY, Palena C. Short-term EGFR blockade enhances immune-mediated cytotoxicity of EGFR mutant lung cancer cells: rationale for combination therapies. Cell Death Dis. 2016;7(9):e2380.

77. David JM, Dominguez C, Palena C. Pharmacological and immunological targeting of tumor mesenchymalization. Pharmacol Ther. 2017;170:212-225.

78. Kusmartsev S, et al. All-trans-retinoic acid eliminates immature myeloid cells from tumor-bearing mice and improves the effect of vaccination. Cancer Res. 2003;63(15):4441-4449. 
79. Akalay I, et al. EMT impairs breast carcinoma cell susceptibility to CTL-mediated lysis through autophagy induction. Autophagy 2013;9(7):1104-1106.

80. Palena C, et al. The human T-box mesodermal transcription factor Brachyury is a candidate target for T-cell-mediated cancer immunotherapy. Clin Cancer Res. 2007;13(8):2471-2478.

81. Tucker JA, et al. Identification and characterization of a cytotoxic T-lymphocyte agonist epitope of brachyury, a transcription factor involved in epithelial to mesenchymal transition and metastasis. Cancer Immunol Immunother. 2014;63(12):1307-1317.

82. Jochems C, et al. Identification and characterization of agonist epitopes of the MUC1-C oncoprotein. Cancer Immunol Immunother. 2014;63(2):161-174 